\title{
Spatial distribution of static stress drops for aftershocks of the 2005 West Off Fukuoka Prefecture earthquake
}

\author{
Yoshihisa Iio $^{1}$, Hiroshi Katao ${ }^{1}$, Tomotake Ueno $^{1}$, Bogdan Enescu ${ }^{1}$, Norio Hirano ${ }^{1}$, Tomomi Okada ${ }^{2}$, Naoki Uchida $^{2}$, \\ Satoshi Matsumoto ${ }^{3}$, Takeshi Matsushima ${ }^{3}$, Kenji Uehira ${ }^{3}$, and Hiroshi Shimizu ${ }^{3}$ \\ ${ }^{1}$ Research Center for Earthquake Prediction, Disaster Prevention Research Institute, Kyoto University, Gokasho Uji 611-0011, Japan \\ ${ }^{2}$ Research Center for Prediction of Earthquakes and Volcanic Eruptions, Graduate School of Science, Tohoku University, \\ Aoba-ku, Sendai, Miyagi 980-8578, Japan \\ ${ }^{3}$ Institute of Seismology and Volcanology, Faculty of Sciences, Kyushu University, Shin' yama, Shimabara, Nagasaki 855-0843, Japan
}

(Received October 15, 2005; Revised June 9, 2006; Accepted June 13, 2006; Online published February 2, 2007)

\begin{abstract}
We investigated the spatial distribution of static stress drops of the aftershocks of the 2005 West Off Fukuoka Prefecture earthquake, with the aim of assessing the possibility that another earthquake will occur on the SE extension of the earthquake fault. The waveforms from six temporary online telemetry stations installed in and around the aftershock region were measured. Small stress drops were estimated for the aftershocks that occurred relatively distant from the SE and NE ends of the earthquake fault. Conversely, the aftershocks that occurred around the SE end of aftershock region are characterized by large stress drops. These results imply the possibility of a stress concentration at the SE edge of the main shock fault.
\end{abstract}

Key words: Stress drop, aftershock, site effect, intraplate earthquake, anelastic attenuation, stress.

\section{Introduction}

The 2005 West Off Fukuoka Prefecture earthquake $(M j=7.0)$ occurred on March 20, 2005 in the GenkaiNada region of the Japan Sea, with aftershocks distributed over about $30 \mathrm{~km}$ in the NW-SE direction (Shimizu et al., 2006). The main shock initiated at $33.739^{\circ} \mathrm{N}, 130.176^{\circ} \mathrm{E}$, at a depth of $9.2 \mathrm{~km}$ (determined by JMA), and its rupture mainly propagated to the southeast (e.g., Asano and Iwata, 2006). The SE end of the aftershock region is located immediately beneath Shikanoshima Island. The metropolitan area of Fukuoka city is located about $10 \mathrm{~km}$ southeast from the SE end of the aftershock area. The quaternary active Kego fault, which runs through Fukuoka city in a NW-SE direction, seems to be located on the southeastward extension of the fault of the 2005 West Off Fukuoka Prefecture earthquake (Research Group for Active Faults of Japan, 1980).

On the basis of the above information, there is a possibility that another large earthquake is impendent on the SE extension of the earthquake fault. Although the successive occurrence of a second large earthquake in the region near a previous large earthquake is a rare event in the intraplate region of Japan (Iio and Kobayashi, 2002), this possibility needs to be evaluated, particularly in the light of the largest aftershock $(M=5.8)$, which occurred around the SE end of the aftershock region on April 20th, with aftershocks that appear to extend toward the Kego fault.

To this end, we investigated the spatial distribution of static stress drops of the aftershocks, using the waveforms

Copyright (c) The Society of Geomagnetism and Earth, Planetary and Space Sciences (SGEPSS); The Seismological Society of Japan; The Volcanological Society of Japan; The Geodetic Society of Japan; The Japanese Society for Planetary Sciences; TERRAPUB from six temporary online telemetry stations installed in and around the aftershock region (Shimizu et al., 2006), in order to assess the possibility of a second large earthquake occurring on the SE extension. Our ultimate goal is to estimate absolute stress around the earthquake fault. We recognize that there is a difference between the obtained static stress drops and the absolute stress. However, a correlation between stress drops and focal depths is indicated by $\mathrm{Ki}$ noshita and Ohike (2002). Further, Ogasawara et al. (2002) have shown that stress drops decrease with decreasing absolute shear stress in a South African gold mine, suggesting that stress drops may be an indicator of the absolute stress.

\section{Data and Methodology}

Figure 1 shows the locations of the temporary online stations at which three-component, short-period $(0.5 \mathrm{~s}$ or $1 \mathrm{~s})$ velocity-type seismometers were installed. The stations were installed immediately above or around the aftershock region. The waveform data, digitized at a rate of $100 \mathrm{~Hz}$ or $200 \mathrm{~Hz}$, were sent through satellite telemetry systems or telephone line networks (Shimizu et al., 2006).

Examples of waveforms of a $M=3.2$ aftershock recorded at all stations are shown in Fig. 2. Observed velocities in the UD component are displayed in absolute and relative scales in the uppermost and middle panels, respectively. In this study, we analyzed the $P$-waves recorded in the UD component. It appears that shapes of the waveforms are different for every station. For example, the waveform recorded at AINS is dominated by low-frequency waves of about $5 \mathrm{~Hz}$, while the low-frequency waves are masked by high frequencies for the waveform at NJHT. Moment-rate spectra are displayed in the lowermost panel after corrections for the response of the seismometer and anelastic at- 


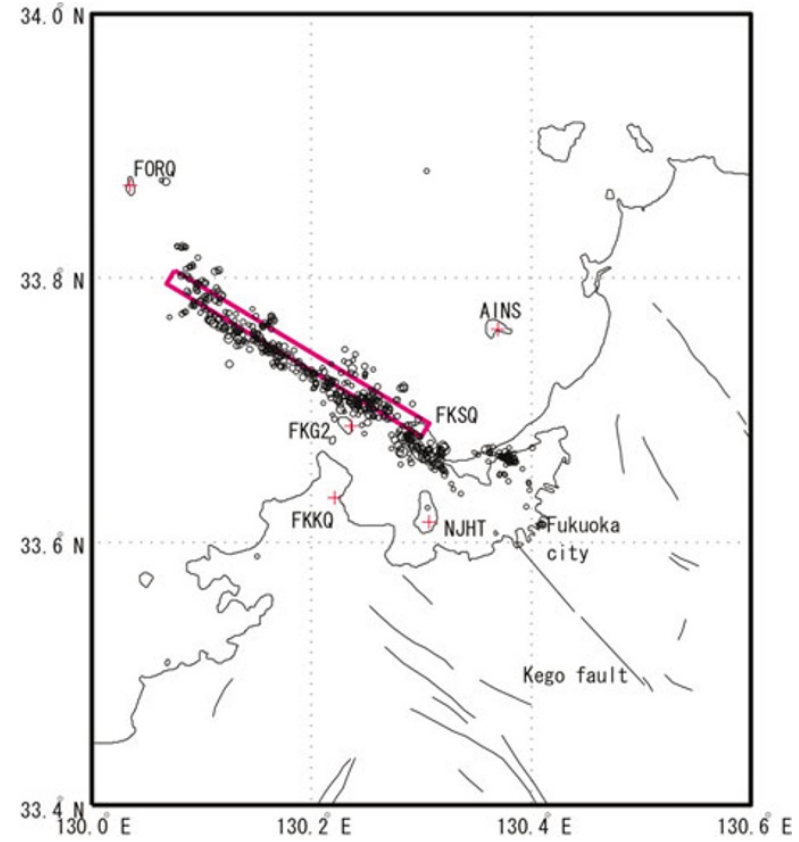

Fig. 1. Locations of the temporary online seismic stations (+) and aftershock distribution. The rectangle indicates the estimated fault plane of the main shock (GSI, 2005).

tenuation (the detailed procedure is described in the following section). The spectra are computed by an FFT using a time window of $2.56 \mathrm{~s}$ around the onset of the $P$-waves, which is adjusted at the center of the time window. The high-frequency peaks are distinct in the spectrum of NJHT. The waveforms at AINS are probably not seriously affected by site response, since the dominant frequencies vary for different aftershocks, while at NJHT the spectral peaks indicate a significant site effect. At FORQ, spectral amplitudes increase with frequency, since ground noises are amplified by the Q correction. FORQ is only located at the NW end of the aftershock region, and average focal distances are longer than those at the others stations. Thus, we do not use the waveform data from FORQ in the following analyses. At the other stations, signal-to-noise ratios (snr) are generally larger than $40 \mathrm{~dB}$, even for $M=2$ aftershocks, since average rms noise amplitudes are fairly small, about $10^{-8}$ to $10^{-7} \mathrm{~m} / \mathrm{s}$.

We need to estimate the site response of each station in order to accurately evaluate the stress drops of aftershocks. Here, we take a new empirical method to cancel the deviation of the source spectrum of each aftershock from the theoretical one by stacking (summing up at each frequency) a large amount of velocity spectra observed at each station (Iio, 2006).

Figure 3 shows velocity spectra of waveforms recorded at each station for the aftershocks with $2 \leq M<4$ that occurred in the central part of the aftershock region (between -5 and $5 \mathrm{~km}$ in the $\mathrm{x}$ direction in Fig. 7) from March 23 to May 31,2005 . The reason why only aftershocks that occurred in the above region were used is that the hypocenters of aftershocks are widely distributed in depth. The spectra are corrected for the response of the seismometer and the effect of anelastic attenuation. The detailed procedure
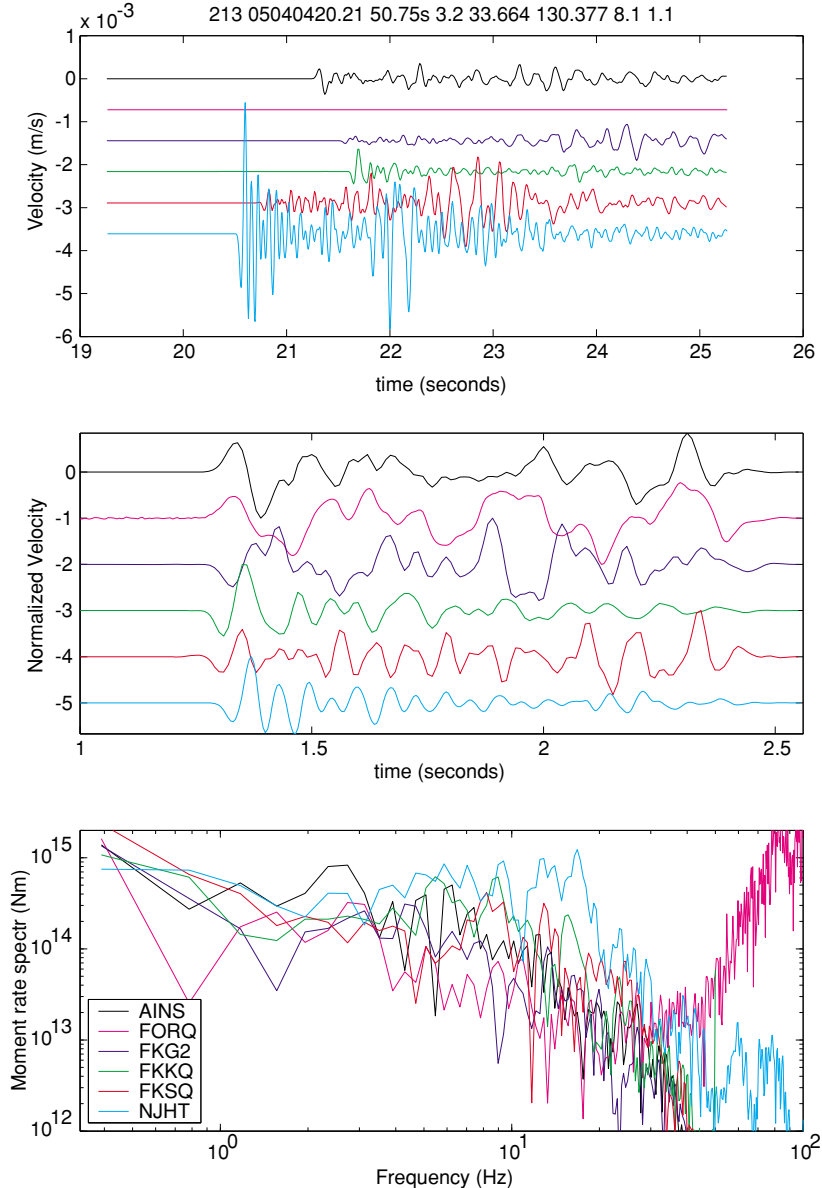

Fig. 2. Examples of waveforms and spectra at each station. Upper, UD components of velocity waveforms; Middle, normalized velocity waveforms aligned at their $P$-wave onsets; Lower, moment-rate spectra after the correction for the seismometer response and anelastic attenuation. Amplitudes of the spectra are modified by Eq. (1). Details are described in the text.

of the correction for anelastic attenuation will be described in a following section. The solid dots in the upper portion of each figure indicate stacked velocity spectra using all of the spectra shown below. A relatively flat spectrum is seen between 5 and $30 \mathrm{~Hz}$ at AINS, while NJHT has large amplitudes at high frequencies.

Dashed lines are the theoretical spectra obtained by stacking velocity spectra at the source, using a cluster of $2 \leq M<4$ earthquakes for which the magnitudefrequency distribution is described by a b-value of 0.75 . The b-value is determined from the observed data. We make the following assumptions in this stacking procedure: (1) each theoretical velocity spectrum is described by the omega-squared model (Boatwright, 1978); (2) the scaling relationship $M_{o} \propto f_{0}^{-3}$ holds, where $f_{0}$ is the corner frequency, and $M_{o}$ is the seismic moment; (3) the corner frequency of a $M=4$ event is $5 \mathrm{~Hz}$, which is estimated from observed spectra with no site response correction and a constant Q (300) correction; (4) the absolute level of the theoretical spectrum is adjusted to the minimum value of the observed stacked velocity spectrum below $5 \mathrm{~Hz}$, since all the temporary stations are installed at hard rock sites and the low frequency amplifications due to multiple reflections 

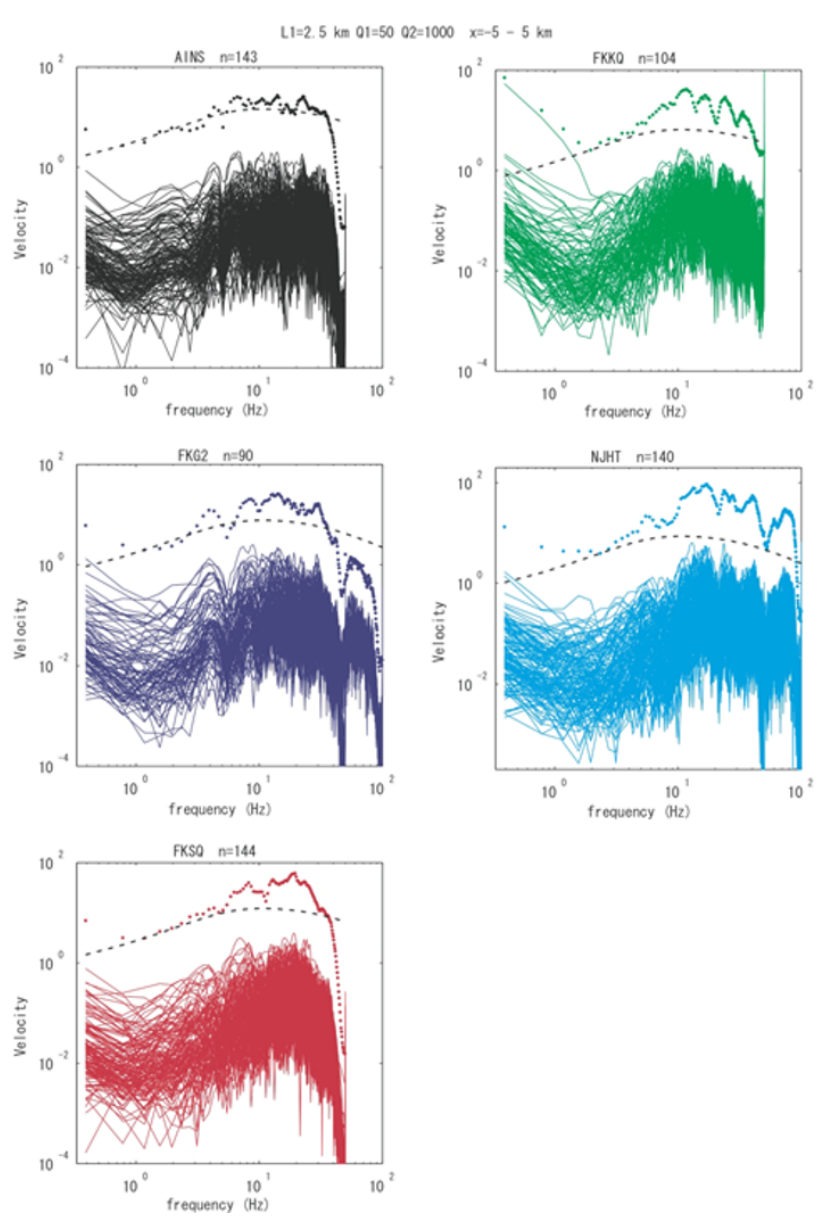

Fig. 3. Estimate of site response. Solid line, Velocity spectra computed at each station for the waveforms of aftershocks with $2 \leq M<4$, which occurred between -5 and $5 \mathrm{~km}$ in the $\mathrm{x}$ direction in Fig. 7. Solid dots, Stacked velocity spectrum using all the spectra shown below; dashed line, Theoretical stacked spectrum. Details are described in the text.

are thought to be small.

The difference between the observed stacked spectrum and the theoretical stacked spectrum directly represents the site response, when the anelastic attenuation is correctly removed. The obtained site responses are shown in the upper panel of Fig. 4. It is noted that these responses include frequency responses of the telemeter systems and also the difference between theoretical (ideal) and actual responses of the seismometers installed.

Anelastic attenuation is corrected assuming a homogeneous two-layered Q structure. Q values are 50 in the top layer of a thickness of $2.5 \mathrm{~km}$ and 1000 below, and are assumed to be independent of frequency.

These $Q$ values are estimated as follows. We assumed that source spectra of the aftershocks of the same magnitude do not vary with depth in the aftershock depth range. In this case, if the path effects (the geometrical spreading and anelastic attenuation) are adequately removed, the corrected spectra at each station do not vary significantly with depth. We then stacked the corrected spectra of aftershock of the same magnitude range but occurring at different depth ranges and compared them with each other, as shown in Fig. 5. Here, we used only the data obtained at AINS and FKKQ because their locations are sensitive to the
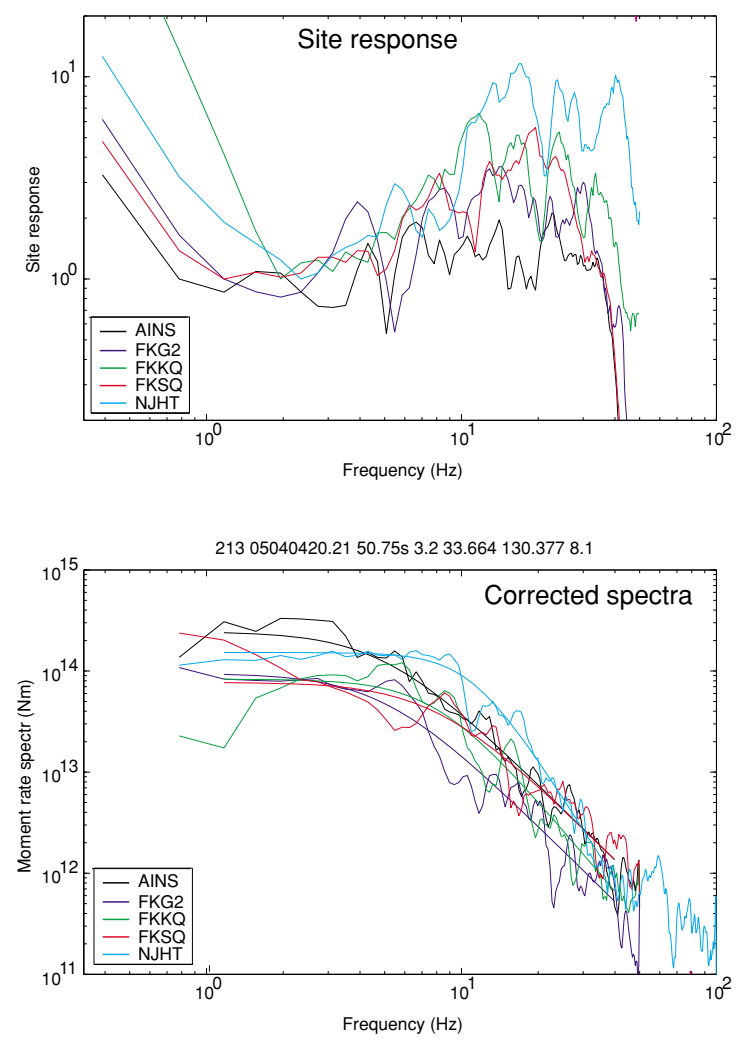

Fig. 4. Upper, site response spectra; Lower, moment-rate spectra after the corrections for anelastic attenuation, site response, and the response of the seismometer. These spectra are modified from those shown in the lowest panel of Fig. 2. The spectra are smoothed with a moving-average of five points.

vertical $\mathrm{Q}$ structure. At the beginning of the $\mathrm{Q}$ analysis, a homogeneous Q structure was assumed; however, we were not able to reproduce similar spectra shapes. We then determined a two-layered Q structure, namely two Q values and a surface layer thickness by trial and error, as stacked spectra for aftershocks occurring in three different depth ranges are similar in shape (Fig. 5). We needed to set a very small $Q$ value for the surface layer with a thickness smaller than the upper limit of the depths of the aftershocks and to set a $Q$ value below that to be very large. These $Q$ values imply that the correction for anelastic attenuation is almost independent of hypocentral depth.

After the corrections for anelastic attenuation, site response and the response of the seismometer, the spectrum $O(f)$ is smoothed with a moving-average of five points and fit to the model spectrum, $O(f)=\frac{\Omega_{0}}{1+\left(f / f_{0}\right)^{p}}$, where $\Omega_{0}$ is the flat level of a displacement spectrum, $f_{0}$ is the corner frequency, and $p$ is the high-frequency fall-off rate. The spectra shown in the lower panel of Fig. 4 are modified from those shown in the lowest panel of Fig. 2. With the site response corrections, the corrected spectra are better fit to the model spectra. The three parameters, $\Omega_{0}, f_{0}$, and $p$, are determined to minimize logarithmic residuals between observed and model spectra by a grid search method. The residuals are computed in a frequency range between 1.2 and $40 \mathrm{~Hz}$, in which the noise levels are low. Consequently, the upper and lower limits of the grid search for $f_{o}$ is set to be 1.0 and $50 \mathrm{~Hz}$, respectively. The seismic moment, $M_{o}$ is 

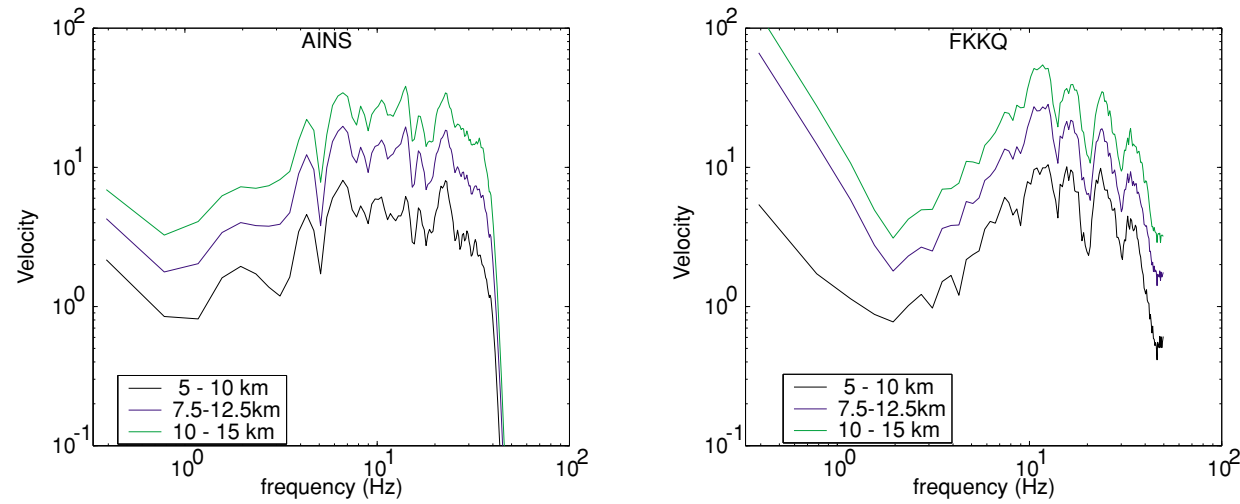

Fig. 5. Comparison of velocity spectra stacked in different depth ranges, for the waveforms of aftershocks with $2 \leq M<4$, that occurred between -5 and $5 \mathrm{~km}$ in the $\mathrm{x}$ direction in Fig. 7.

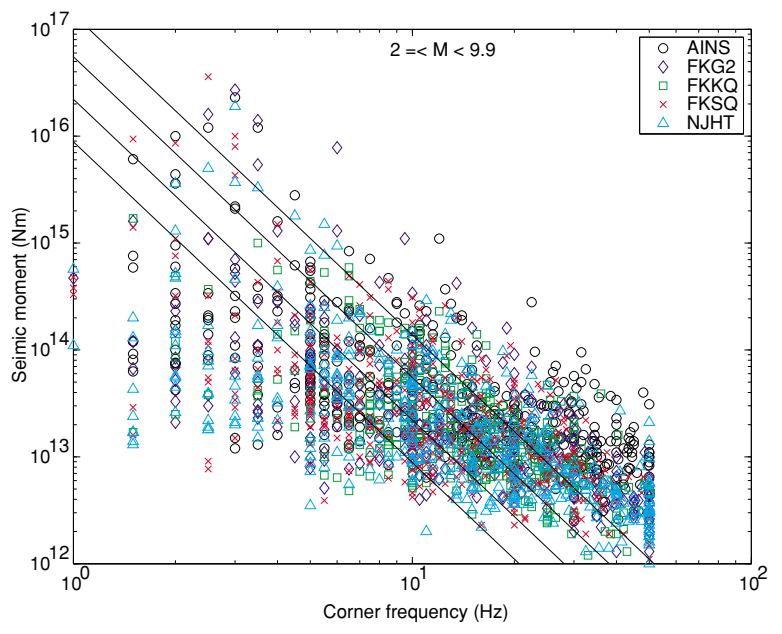

Fig. 6. The relationship between $M_{o}$ and $f_{0}$ for each station.

computed from the equation,

$$
M o=4 \pi \rho l V p^{3} \frac{\Omega_{0}}{F R_{\theta \varphi}}(\mathrm{Nm})
$$

following Hanks and Wyss (1972), whereis the density $\left(2.7 \times 10^{3} \mathrm{~kg} / \mathrm{m}^{3}\right), l$ is the distance $(\mathrm{m}), V p$ is the $P$ wave velocity $\left(6 \times 10^{3} \mathrm{~m} / \mathrm{s}\right), F$ is the free surface correction (2), and $R_{\theta \varphi}$ is the average radiation pattern (0.4). The stress drop is computed as $\Delta \sigma=\left(\frac{7}{16}\right) \frac{M_{0}}{r^{3}}(\mathrm{MPa})$, where $r$ is the source radius ( $\mathrm{m}$ ) computed as $r=\frac{2.34 \mathrm{Vp}}{2 \pi f_{0}}$ (Hanks and Wyss, 1972). The correction for radiation pattern is not used, since fault plane solutions are not available for small events.

\section{Results}

We analyzed 514 aftershocks with $M \leq 2.0$ that occurred from March 23 to May 31, 2005. The relation between $M_{o}$ and $f_{0}$ is shown in Fig. 6. Plotted data are widely scattered. As shown in the following, this is mainly due to variation in source spectra, but errors in spectral fitting also affect the scatter. The scaling relationship $M_{o} \propto f_{0}^{-3}$ seems to hold for these data.

A stress drop is first computed at every station-with the exception of FORQ - for each aftershock. The values of stress drops are then logarithmically averaged, using only the aftershocks for which stress drops were determined at more than three stations.

The spatial distribution of the static stress drops is shown in Fig. 7 for the aftershocks with $2.3 \leq M<3.5$ and standard errors in logarithmic stress drop smaller than 0.5 . The magnitude limits are set to avoid the problems that can result from small snr, the high frequency limit of the analysis, and size-dependent stress drop changes. About $10 \%$ of the data are excluded by the limits of the standard errors. The general pattern, as shown in Fig. 7, does not change even if all the data are plotted. It can be seen that $60 \%$ of the calculated stress drops have a standard error smaller than 0.3 (a factor 2 in a linear scale). In Fig. 7, stress drops are separated into five groups, for which the values differ by a factor of 3 . Small stress drops are estimated for the aftershocks that occurred relatively distant from the SE and NW ends of the earthquake fault. Stress drops are also small for the aftershocks that occurred on the NE side ( $Y \geq 2$ in Fig. 7) of the estimated fault plane of the main shock. These aftershocks are likely located outside of the fault zone of the main shock, since it is inferred from the hypocentral distributions of secondary aftershocks (aftershocks of a large aftershock) that relative hypocentral errors are at most $2 \mathrm{~km}$ in horizontal directions. These results indicate that off-fault events are characterized by small stress drops.

For those aftershocks that occurred on or close to the estimated earthquake fault, there is a tendency that stress drops are small near the upper bound of the aftershock distribution. The secondary aftershocks of the $M=5.8$ event on April 20, which occurred near the SE end of the aftershock region, are characterized by large stress drops. In the region of the 'asperity' $(-10<X<-5)$, where the slip of the main shock is larger (e.g., Asano and Iwata, 2006), it appears that stress drops can be slightly smaller than those in the surrounding region.

\section{Discussion and Concluding Remarks}

First, we will assess errors in the estimates of stress drops. The rms residuals of the spectral model function do not show a distinct local minimum but, rather, a relatively smooth shape. Thus, the spectral parameters for each sta- 

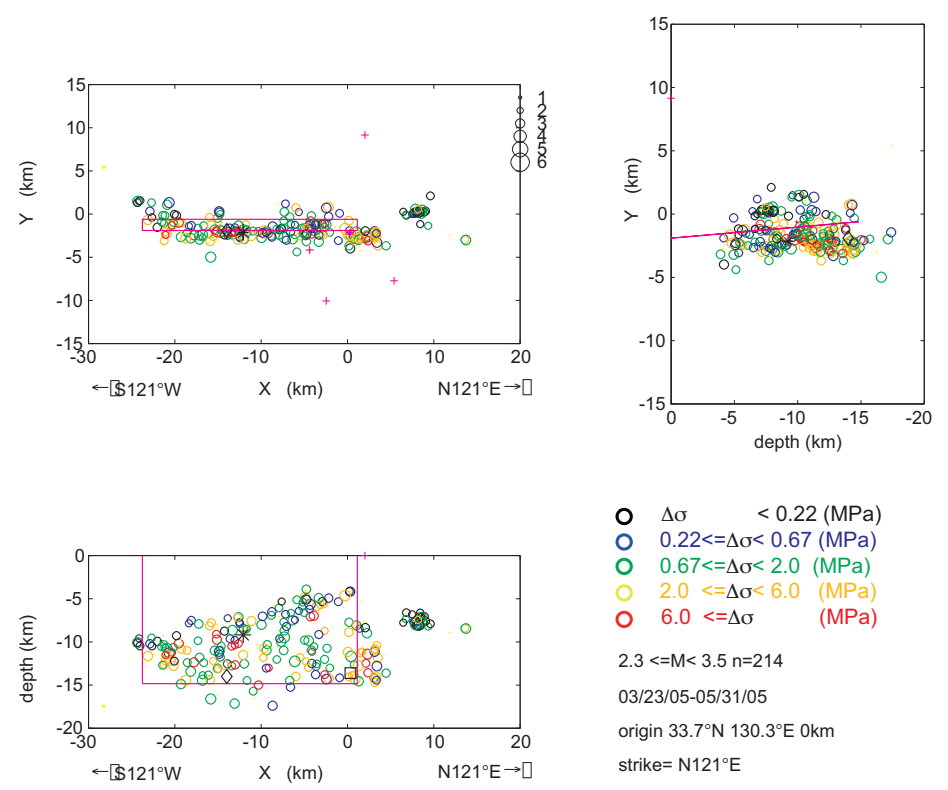

Fig. 7. The spatial distribution of static stress drops of the aftershocks with $2.3 \leq M<3.5$ and for which the logarithm of the standard errors in stress drop are smaller than 0.5 , projected in the horizontal and two vertical planes. Colors correspond to the magnitude of stress drops: red, $>6.0 \mathrm{MPa}$; yellow, 2.0-6.0 MPa; green, 0.67-2.0 MPa; blue, 0.22-0.67 MPa; black, <0.22 MPa. The star and diamond indicate the hypocenter of the mainshock determined by JMA and Kyushu University, respectively. The open square is the hypocenter of the largest aftershock $(M=5.8)$. The rectangle indicates the estimated fault plane of the main shock (GSI, 2005).

tion are thought to be accurately determined. The problem is their differences between stations. The maximum difference between stations is about one order of magnitude, and probably results from the correction of the site responses. Although the single site response correction is applied to all data, it is possible that site responses could differ, depending on the back-azimuths of aftershocks. Furthermore, we do not consider errors caused by complicated spectral shapes, such as a secondary high-frequency peak in the velocity spectra.

Our most important results are: (1) the stress drops of the aftershocks that occurred relatively distant from the earthquake fault are small, and (2) the stress drops for the aftershocks of the $M=5.8$ (April, 20) that occurred near the SE end of the aftershock region are large. The first implies that off-fault aftershocks are generated on very weak pre-existing fault planes. These are probably not related to the stress concentration that possibly leads to another large earthquake. On the other hand, the second result suggests the possibility that a stress concentration occurs at the SE end of the main shock fault. We need to investigate this possibility, for example, by studying the temporal and spatial changes in seismicity and focal mechanisms.

Acknowledgments. We are grateful to the group for the urgent joint observation of the 2005 west off Fukuoka prefecture earthquake for providing us the temporary online earthquake data. We utilized $P$-wave arrival times and hypocentral data from the JMA preliminary catalog. The authors thank James Mori Jiro for his critical review of the manuscript. We are grateful to the teachers and staff of the Ainoshima elementary school for their great help in installation and maintenance. We also thank Masaki Saito, Miho Hori, Yuki Kono, Atsushi Watanabe, for their contributions to the seismic observation. We thank Charles Mueller and another anonymous reviewer for their constructive comments.

\section{References}

Asano, K. and T. Iwata, Source process and near-source ground motions of the 2005 West Off Fukuoka Prefecture earthquake, Earth Planets Space, 58, 93-98, 2006.

Boatwright, J., Detailed spectral analysis of two small New York state earthquakes, Bull. Seismol. Soc. Am., 68, 1177-1131, 1978.

Geographical Survey Institute, Earthquake: Mj7.0 in West-off Fukuoka pref. (Northern Kyushu), on 20 March 2005, http://www.jishin.go.jp/ main/chousa/05mar_fukuoka/p17.gif, 2005.

Hanks, T. C. and M. Wyss, The use of body wave spectra in the determination of seismic source parameters, Bull. Seismol. Soc. Am., 62, 561-569, 1972.

Iio, Y., A new empirical method to estimate the site response by stacking observed velocity waveforms, 2006 (in preparation).

Iio, Y. and Y. Kobayashi, A physical understanding of large intraplate earthquakes, Earth Planets Space, 54, 1001-1004, 2002.

Jin, A. and E. Fukuyama, Seismic Energy for Shallow Earthquakes in Southwest Japan, Bull. Seismolog. Soc. Am., 95, 1314-1333, 2005.

Kinoshita, S. and M. Ohike, Scaling Relations of Earthquakes That Occurred in the Upper Part of the Philippine Sea Plate beneath the Kanto Region, Japan, Estimated by Means of Borehole Recordings, Bull. Seismol. Soc. Am., 92(2), 611-624, DOI: 10.1785/0120010134, 2002.

Ogasawara, H., S. Sato, S. Nishi, H. Kawakata, and the research group for Semi-controlled earthquake-generation experiments in South African deep gold mines, Temporal variation of seismic parameters associated with an Mw2 event monitored at a 100-200 m distance, Seismogenic Process Monitoring, 173-184, Balkema, Rotterdam, 2002.

Research Group for Active Faults of Japan, Active faults in Japan, sheet maps and inventories, Univ. of Tokyo Press, Tokyo, 1980.

Shimizu, H., H. Takahashi, T. Okada, T. Kanazawa, Y. Iio, H. Miyamachi, T. Matsushima, M. Ichiyanagi, N. Uchida, T. Iwasaki, H. Katao, K. Goto, S. Matsumoto, N. Hirata, S. Nakao, K. Uehira, M. Shinohara, H. Yakiwara, N. Kame, T. Urabe, N. Matsuwo, T. Yamada, A. Watanabe, K. Nakahigashi, B. Enescu, K. Uchida, S. Hashimoto, S. Hirano, T. Yagi, Y. Kohno, T. Ueno, M. Saito, and M. Hori, Aftershock seismicity and fault structure of the 2005 West Off Fukuoka Prefecture Earthquake ( $\left.M_{\text {JMA }} 7.0\right)$ derived from urgent joint observations, Earth Planets Space, 58, this issue, 1599-1604, 2006.

Y. Iio (e-mail: iio@rcep.dpri.kyoto-u.ac.jp), H. Katao, T. Ueno, B. Enescu, N. Hirano, T. Okada, N. Uchida, S. Matsumoto, T. Matsushima, K. Uehira, and H. Shimizu 